\title{
ATRATIVIDADE E PREFERÊNCIA ALIMENTAR DE Epicauta atomaria (Ger.) EM ALGUMAS ESPÉCIES DE MARACUJÁ ${ }^{1}$
}

\author{
ARLINDO LEAL BOIÇA JUNIOR², JAIME ZUCCOLOTTO BAPTISTA², JOÃO CARLOS DE OLIVEIRA ${ }^{3}$ \\ FLÁVIO GONÇALVES DE JESUS ${ }^{2}$
}

RESUMO: Avaliou-se, em laboratório, a não-preferência para alimentação de Epicauta atomaria em diferentes espécies de maracuja, em teste com e sem chance de escolha. Verificou-se a melhor densidade de insetos que proporcionam a discriminação quanto aos graus de resistência. Para avaliação, utilizaram $P$. setacea, $P$. alata, $P$. edulis, $P$. cincinnata e $P$. laurifolia. Para os testes com e sem chance de escolha, a não-preferência para alimentação foi avaliada através da atratividade, onde foi contado o número de insetos atraídos por espécie, em placa de Petri. As espécies $P$. laurifolia e $P$. alata foram resistentes a $E$. atomaria, expressando o tipo de resistência à nãopreferência para alimentação. As densidades de E. atomaria que melhor discriminaram as espécies de maracujazeiro, são três e cinco (teste com chance de escolha) e dois (teste sem chance de escolha). P. edulis, P. setacea e P. cincinnata são suscetíveis a E. atomaria. Termos para indexação: maracujá, resistência de plantas, tipo de resistência.

\section{ATTRACTIVITY AND FEEDING PREFERENCE OF Epicauta atomaria (Ger.) IN SOME PASSION FRUIT SPECIES}

ABSTRACT-The no preference for feeding of Epicauta in different species of passion fruit in free choice and no choice tests was evaluated under laboratory conditions. It was verified the best density of insects that provides the discrimination of resistance degrees. P. setacea, P. alata, P. edulis, P. cincinnata and P. laurifólia were evaluated. For the tests with and without possibility of choice, the preference for no feeding was evaluated through the attractiveness, where the number of insects attracted by species in Petri dishes was counted. The species $P$. laurifolia e P. alata had been resistant to E. atomaria, expressing the type of resistance of no preference for feeding. The densities of E. atomaria that better discriminated the species of passion fruit are three and five (test with and without choice) and two (test without possibility of choice) P. edulis, P. setacea e P. cincinnata are susceptible to E. atomaria.

Index Terms: passion fruit, host plant resistance, type of resistance.

\section{INTRODUÇÃO}

O maracujazeiro é originário das regiões tropicais, encontrando no Brasil condições excelentes para o seu cultivo, tornando-se o maior produtor mundial, com uma produção de 485.342 toneladas, sendo destas 124.443 toneladas produzidas na região Sudeste, apresentando o Estado de São Paulo uma produção de 79.527 toneladas de frutos (Agrianual, 2006).

Entretanto, essa cultura vem sofrendo grandes problemas agronômicos para se desenvolver, principalmente em relação a pragas e doenças. Dentre as mais prejudiciais, são as lagartas desfolhadoras e percevejos sugadores, considerados mais freqüentes e severas nas principais regiões produtoras de maracujá no Brasil (Ruggiero et al., 1996; Boiça Junior, 1998).

Boaretto \& Brandão (1994) alertaram para o surgimento de determinadas espécies de insetos assumindo o "status" de praga-chave e sobrepondo em danos aquelas tradicionalmente relacionadas na literatura, como a Epicauta atomaria (Germar,
1821) (Coleoptera:Meloidae). Essa é uma praga polífaga que se alimenta do maracujazeiro, da batata, do tomate e de outras solanáceas e plantas hortículas. As posturas são feitas normalmente no solo; porém, quando o nível populacional é muito alto, pode colocar ovos na face inferior das folhas. Cada fêmea oviposita em média 120 ovos, que ficam aderentes uns aos outros; dez dias após a postura, ocorre a eclosão dos ovos; as larvas possuem cabeça grande, pernas alongadas e transformam-se em pupas no solo. O adulto é um besouro com cerca de 8 a $17 \mathrm{~mm}$ de comprimento e de coloração preta (Gallo et al., 2002).

Os principais danos consistem na destruição das folhas em muito pouco tempo, deixando-as reduzidas a nervuras (Boiça Junior, 1998). Segundo Rodrigues Neto \& Guilherme (2000), as principais infestações ocorrem em reboleiras, inicialmente nas bordas dos pomares, onde grande número de adultos se alimentam vorazmente do maracujazeiro, reduzindo consideravelmente sua área fotossintética.

O método de controle convencional mais utilizado pelos

'(Trabalho 196-06). Recebido em: 27-11-2006. Aceito para publicação em: 30-07-2007.

${ }^{2}$ Departamento de Fitossanidade da Faculdade de Ciências Agrárias e Veterinárias/UNESP, Câmpus de Jaboticabal. Via de acesso Prof. Paulo Donato Castellane, Km 5 14884-900. Jaboticabal-SP. aboicajr@fcav.unesp.br

${ }^{3}$ Departamento de Produção Vegetal, da Faculdade de Ciências Agrárias e Veterinárias/UNESP, Câmpus de Jaboticabal. Via de acesso Prof. Paulo Donato Castellane, Km 5 14884-900. Jaboticabal-SP. 
agricultores, para minimizar os danos causados por essas pragas, é através do uso de produtos químicos. Porém, segundo Fancelli (1992), a utilização desses produtos pode aumentar a incidência de mortalidade dos inimigos naturais e dos insetos polinizadores.

Outro método de controle dessa praga seria o uso de espécies resistentes, o qual é considerado o ideal, pois trata-se de um método que mantém a população da praga abaixo dos níveis de danos econômicos sem causar nenhum distúrbio ou poluição ao ecossistema e sem provocar qualquer ônus ao agricultor (Lara, 1991).

As pesquisas, tanto no Brasil como no mundo, são muito restritas nessa área. Boiça Junior et al. (1996) discriminaram espécies de maracujazeiro, em laboratório, verificando a preferência alimentar de E. atomaria. A espécie preferida pelos insetos foi Passiflora caerulea, enquanto as de menor preferência foram $P$. setacea, $P$. cincinnata, $P$. alata, $P$. coccinea, $P$. nitida, $P$. edulis, híbrido (P.alata $\mathrm{x}$ P.macrocarpa) e o híbrido (P. edulis $\mathrm{x} P$. alata).

O gênero Passiflora é o maior da família, com cerca de 400 espécies. No Brasil, ocorrem 110 espécies, o que traz ampla variabilidade natural, com possibilidades de busca na resistência a essa praga. Porém existem poucos trabalhos em relação a este assunto. Assim, o objetivo deste trabalho foi avaliar a nãopreferência para alimentação de E. atomaria por diferentes espécies de maracujazeiro, em teste com e sem chance de escolha, bem como determinar a densidade de adultos da praga que melhor discriminem as espécies quanto aos graus de resistência.

\section{MATERIAL E MÉTODOS}

Os testes de não-preferência para alimentação foram conduzidos no Laboratório de Resistência de Plantas a Insetos do Departamento de Fitossanidade da Faculdade de Ciências Agrárias e Veterinárias, UNESP, Jaboticabal-SP, entre os anos de 2002 e 2003. Os insetos utilizados nos testes foram coletados em plantas de maracujazeiro e plantas de pimenta (Capsicum spp.)

Para evitar o condicionamento pré-marginal citado por Lara (1991), os insetos de E. atomaria foram colocados por sete dias em recipiente com caruru (Amaranthus deflexus L.) e 4 horas antes do início do ensaio não foram alimentados.

Como recipiente, foram utilizadas placas de Petri (diâmetro de $15 \mathrm{~cm}$ ) com fundo revestido de papel-filtro umedecido. Os ensaios foram conduzidos à temperatura de $25 \pm 1{ }^{\circ} \mathrm{C}$, umidade relativa de $60 \pm 10 \%$ e fotofase de 14 horas.

Nos ensaios de consumo foliar, foi utilizado um vazador com o intuito de cortar os discos de folhas novas com um diâmetro de 3,2 cm. Cada genótipo foi representado por dois círculos foliares com uma área correspondente de $8,042 \mathrm{~cm}^{2}$. O tempo de consumo analisado, tanto para o teste com chance de escolha como para o teste sem chance de escolha, foi de 24 horas.

Para o teste de consumo foliar de cada espécie, foram retirados discos aos pares nas folhas, simetricamente opostos em relação à nervura central. Logo após o final dos testes as partes restantes dos discos foram secas em estufas a $63 \pm 3{ }^{\circ} \mathrm{C}$ e, pela diferença de massa seca dos discos foliares da alíquota, determinou-se a massa seca consumida em grama de cada genótipo avaliado.

Os dados obtidos foram transformados em $(x+0,5)^{1 / 2} \mathrm{e}$ submetidos à análise de variância, pelo teste de Fischer, enquanto as médias foram comparadas pelo teste de Tukey, a 5\% de probabilidade.

Teste com chance de escolha. Os insetos foram liberados no centro das placas com o propósito de ficarem eqüidistantes das áreas dos discos das folhas. Foram utilizadas 5 espécies por placa de Petri: P. setacea, P. alata, P. edulis, $P$. cincinnata e $P$. laurifolia. Cada genótipo foi submetido a quatro números de $E$. atomaria liberados por placas, correspondendo a $0 ; 1 ; 3$ e 5 insetos adultos, com o propósito de verificar qual o número que melhor discrimine as espécies testadas. Foram realizadas 10 repetições para cada tratamento em blocos ao acaso, dispostos em esquema fatorial 5 (genótipos) x 4 (número de insetos).

Foi observada a atratividade dos adultos de E. atomaria a $1 ; 3 ; 5 ; 10 ; 15 ; 30 ; 60$ e 120 minutos após a liberação.

Teste sem chance de escolha. Foram utilizadas duas espécies de maracujá, $P$. laurifolia e $P$. edulis, que foram selecionadas no teste anterior, sendo representadas por dois discos foliares, com uma área correspondente a $8,042 \mathrm{~cm}^{2}$ cada disco. Foi variado o número de E. atomaria liberado em cada placa de Petri contendo o genótipo resistente ou suscetível, com $0 ; 1 \mathrm{e} 2$ insetos adultos. Para cada tratamento, foram realizadas 10 repetições em blocos ao acaso, dispostos em esquema fatorial 2 (genótipos) x 3 (número de insetos)

Nos testes de atratividade, foram realizadas avaliações do número de E. atomaria atraídas em cada disco a: $1 ; 3 ; 5 ; 10 ; 15 ; 30$ e 120 minutos após a liberação dos insetos na placa de Petri.

\section{RESULTADOS E DISCUSSÃO}

Analisando-se a atratividade de E. atomaria (Tabela 1), observa-se que, dentre os genótipos, aos 3; 5 e 10 minutos, ocorreu menor preferência dos insetos por P. laurifolia e $P$. alata, diferenciando a maior atratividade por $P$. edulis, $P$. setacea e $P$. cincinnata. Aos 15 minutos (Tabela 1), o genótipo com menor número de insetos foi P. laurifolia e com maior número de insetos destacaram-se $P$. setacea e $P$. cincinnata. É interessante salientar que, após esse período, pode estar envolvida a presença de outros estímulos, que não o atraente/repelente, dentro da cadeia, como a deterência (Lara, 1991); no entanto, de modo geral, nota-se que $P$. laurifolia e $P$. alata continuaram com baixa preferência pelo inseto.

Nas avaliações de 1 a 60 minutos (Tabelas 1 e 3), a densidade de 3 e de 5 insetos diferiu das demais, proporcionando maior número de insetos atrativos; porém, aos 120 minutos, apenas a de 5 insetos. Esses resultados caracterizam o efeito gregário que a espécie apresenta em condições de campo (Gallo et al., 2002). Para as interações de genótipos por insetos (Tabela 1), nota-se que, nas avaliações de 1; 30 e 60 minutos, não se observaram diferenças significativas; porém, nas demais avaliações, as interações foram significativas a $1 \%$ de probabilidade.

No intervalo de avaliação de 1 a 15 minutos (Tabela 2), em relação às espécies, nota-se que $P$. laurifolia foi a menos atrativa, quando comparada a $P$. setacea, $P$. edulis e $P$. cincinnata, que 
sofreram maior presença da E. atomaria. Em todo intervalo da avaliação de 1 a 120 minutos (Tabela 2), os genótipos menos atrativos foram o $P$. alata e o P. laurifolia, indicando a possível presença de algumas substâncias repelentes e ou deterrentes a E. atomaria. As espécies mais atrativas foram $P$. setacea, P. edulis e $P$. cincinnata. Em relação à densidade populacional na avaliação de 1 a 15 minutos, a densidade de 1 inseto diferiu da densidade de 3 a 5 insetos, demonstrando o efeito gregário de E. atomaria em maracujazeiro, concordando com o relato de Gallo et al. (2002).

Por outro lado, no intervalo de 1 a 120 minutos (Tabela 2), as diferenças entre as densidades populacionais de E. atomaria foram significativas, sendo que a densidade de 5 insetos influenciou em maior atratividade em relação ao de 1 e de 3 insetos.

A interação entre os genótipos das diferentes espécies versus insetos foi significativa nos dois intervalos de avaliação da atratividade (1 minuto a 15 minutos e de 1 minuto a 120 minutos).

Analisando o desdobramento das interações (Tabela 3), constata-se, no geral, para o efeito de diferentes espécies dentro de densidade de insetos, que ocorreram diferenças estatísticas significativas nas densidades de 3 e 5 insetos por placas, onde os menores números foram observados em $P$. laurifolia e $P$. alata, caracterizando o menor interesse dos insetos por essas espécies.

Para efeito da densidade de insetos dentro das espécies (Tabela 3), verifica-se, no geral, que ocorreram diferenças significativas para $P$. setacea e $P$. cincinnata, com maior número de insetos atraídos nas densidades de 3 e de 5 por placa, o que sugere o efeito gregário dos mesmos.

Quanto à Tabela 4, nota-se que ocorreram diferenças significativas em relação às espécies de maracujazeiros avaliados e não ocorreram diferenças em relação à densidade do número de E. atomaria, havendo menor consumo de folhas de P. laurifolia, $P$. cincinnata e $P$. alata, e sugerindo apresentarem resistência do tipo não-preferência para alimentação, quando comparados com as demais espécies. Com esse mesmo propósito, Boiça Junior et al. (1996) e Baldin \& Lara (2002) verificaram que discos foliares de $P$. alata e $P$. cincinnata, para os primeiros autores, e $P$. alata para os segundos, foram pouco consumidos pelas vaquinhas. Em outro trabalho, porém em condições de campo, Baptista et al. (2005) relacionam, dentre outros, P.alata e P. laurifolia como resistentes

\section{a E. atomaria.}

Na densidade populacional de E. atomaria (Tabela 4), não ocorreram diferenças significativas quando foram comparados 1 ; 3 e 5 insetos por placa de Petri, ou seja, não ocorreu aumento de consumo em relação ao número de insetos utilizados.

No teste sem chance de escolha, as avaliações de 1 e 3 minutos (Tabela 5), na espécie $P$. edulis, proporcionaram maior atração pelo inseto que $P$. laurifolia. Neste período de avaliação, observou-se grande movimentação dos insetos sobre essas espécies.

Aos 15 minutos e nas avaliações seguintes (Tabela 5), $P$. edulis continuou sendo mais atrativo que $P$. laurifolia, porém nota-se que, a partir dos 15 minutos que os insetos pararam de movimentar-se e iniciaram suas primeiras mordidas de prova em P. edulis e P. laurifolia , ficaram em sua maioria na borda da placa de Petri, rejeitando esse genótipo.

Para a densidade de E. atomaria (Tabela 5), nota-se que não ocorreram diferenças significativas em relação aos intervalos de avaliação.

Pela análise de desdobramento da interação na avaliação de 120 minutos (Tabela 6), constatam-se diferenças significativas de densidade de insetos dentro de $P$. edulis, onde o maior número de insetos atraídos ocorreu na densidade de 2 insetos. Para efeito de genótipos dentro da densidade, verificam-se diferenças a um $\mathrm{e}$ dois insetos, onde o P. laurifolia foi sempre o menos atrativo.

Nos intervalos de avaliação de 1 e 15 minutos e de 1 a 120 minutos (Tabela 7), a espécie $P$. edulis foi mais atrativa que $P$. laurifolia. Quanto à densidade populacional de E. atomaria, tanto 1 como 2 insetos não mostraram diferenças significativas. As interações não diferiram entre espécies versus insetos nestes dois intervalos de avaliação.

No teste sem chance de escolha para o consumo foliar (Tabela 8), P. edulis diferiu estatisticamente de P. laurifolia. Constata-se que a espécie $P$. laurifolia exerceu a resistência do tipo não-preferência para alimentação a $E$. atomaria, e também se observa que $P$. laurifolia apresentou alguma substância repelente e/ou deterrente à praga. Não ocorreram diferenças significativas tanto para a densidade de 1 como para 2 insetos por placa de Petri.

TABELA 1 - Número médio de adultos de Epicauta atomaria atraídos por discos foliares de cinco especies de maracujazeiro, em três densidades de adultos (teste com chance de escolha). Jaboticabal- SP. 2002-2003.

\begin{tabular}{|c|c|c|c|c|c|c|c|c|}
\hline \multirow[t]{2}{*}{ Espécies (E) } & \multicolumn{8}{|c|}{ Número de insetos atraidos } \\
\hline & 1 minuto & 3 minutos & 5 minutos & 10 minutos & 15 minutos & 30 minutos & 60 minutos & 120 minutos \\
\hline P. setacea & $0,85 \mathrm{ab}$ & $0,93 \mathrm{ab}$ & $1,01 \mathrm{a}$ & $1,04 \mathrm{a}$ & $0,99 \mathrm{a}$ & $1,08 \mathrm{a}$ & $1,01 \mathrm{a}$ & $1,12 \mathrm{a}$ \\
\hline P. alata & $0,77 \mathrm{ab}$ & $0,77 \mathrm{bc}$ & $0,77 \mathrm{c}$ & $0,75 \mathrm{c}$ & $0,86 \mathrm{ab}$ & $0,79 \mathrm{~b}$ & $0,79 \mathrm{bc}$ & $0,70 \mathrm{~b}$ \\
\hline P. edulis & $0,87 a b$ & $0,96 \mathrm{a}$ & $1,04 \mathrm{a}$ & $0,98 \mathrm{a}$ & $0,93 \mathrm{ab}$ & $0,98 \mathrm{a}$ & $0,96 \mathrm{ab}$ & 0,99 a \\
\hline P. cincinnata & $0,95 \mathrm{a}$ & $0,93 \mathrm{ab}$ & $0,97 \mathrm{ab}$ & $0,98 \mathrm{a}$ & $0,95 \mathrm{a}$ & $0,99 \mathrm{a}$ & $1,02 \mathrm{a}$ & $0,96 \mathrm{a}$ \\
\hline P. laurifolia & $0,75 \mathrm{a}$ & $0,75 \mathrm{c}$ & $0,79 \mathrm{bc}$ & $0,77 \mathrm{~b}$ & $0,74 \mathrm{~b}$ & $0,73 \mathrm{~b}$ & $0,74 \mathrm{c}$ & $0,72 \mathrm{c}$ \\
\hline$F(E)$ & $2,71^{*}$ & $4,73^{* *}$ & $6,42^{* *}$ & $6,96^{* 4}$ & $3.55^{* 4}$ & $9,19^{* *}$ & $5,69^{* *}$ & $6,55^{* *}$ \\
\hline Insetos (I) & 1 minuto & 3 minutos & 5 minutos & 10 minutos & 15 minutos & 30 minutos & 60 minutos & 120 minutos \\
\hline Um inseto & $0,74 \mathrm{~b}$ & $0,76 \mathrm{~b}$ & $0,80 \mathrm{~b}$ & $0,78 \mathrm{~b}$ & $0,76 \mathrm{~b}$ & $0,78 \mathrm{~b}$ & $0,77 \mathrm{~b}$ & $0,77 b$ \\
\hline Três insetos & $0,88 \mathrm{a}$ & $0,90 \mathrm{a}$ & $0,95 \mathrm{a}$ & $0,90 \mathrm{ab}$ & $0,91 \mathrm{a}$ & $0,92 \mathrm{a}$ & $0,93 \mathrm{a}$ & $0,89 \mathrm{~b}$ \\
\hline Cinco insetos & $0,89 \mathrm{a}$ & $0,94 \mathrm{a}$ & $1,01 \mathrm{a}$ & $1,03 \mathrm{a}$ & $1,01 \mathrm{a}$ & $1,04 \mathrm{a}$ & $1,00 \mathrm{a}$ & $1,03 \mathrm{a}$ \\
\hline F (I) & $4,90^{* *}$ & $7,15^{* *}$ & $7,8^{* *}$ & $6,8^{* *}$ & $8,80^{* *}$ & $11,06^{* *}$ & $7,5^{* *}$ & $11,58^{* *}$ \\
\hline$F(E \times I)$ & $0,71^{\mathrm{NS}}$ & $2,85^{* *}$ & $2,53^{* *}$ & $2,98^{* *}$ & $2,17^{\circ *}$ & $1,10^{\mathrm{NS}}$ & $0,59^{\mathrm{NS}}$ & $4,10^{* *}$ \\
\hline D. M. S. & 0,25 & 0,24 & 0,27 & 0,27 & 0,29 & 0,26 & 0,30 & 0,26 \\
\hline C. V. & 30,82 & 28,24 & 29,93 & 30,08 & 32,37 & 28,97 & 33,09 & 28,93 \\
\hline
\end{tabular}

${ }^{1}$ Médias seguidas da mesma letra não diferem estatisticamente entre si na coluna, pelo teste de Tukey, ao nível de $5 \%$ de probabilidade. (Para análise, os dados foram transformados em $\left.(\mathrm{x}+0,5){ }^{1 / 2}\right)$. ${ }^{* *}$ Significativo a $1 \%$ de probabilidade. ${ }^{*}$ Significativo a $5 \%$ de probabilidade. Ns Nãosignificativo. 
TABELA 2 - Número médio de adultos de Epicauta atomaria atraídos de 1 a 15 minutos e de 1 a 120 minutos, por discos foliares, de cinco espécies de maracujazeiro, em três densidades de adultos em teste com chance de escolha. Jaboticabal- SP. 20022003.

\begin{tabular}{lll}
\hline Espécies (E) & \multicolumn{1}{c}{ Número de ihsetos atraídos } \\
\hline & 1 minuto a 15 minutos & 1 minuto a 120 minutos \\
\cline { 2 - 3 } P. setacea & $1,65 \mathrm{a}$ & $2,17 \mathrm{a}$ \\
P. alata & $1,07 \mathrm{bc}$ & $1,17 \mathrm{~b}$ \\
P. edulis & $1,49 \mathrm{a}$ & $1,95 \mathrm{a}$ \\
P. cincinnata & $1,44 \mathrm{a}$ & $1,92 \mathrm{a}$ \\
P. laurifolia & $0,90 \mathrm{c}$ & $0,96 \mathrm{~b}$ \\
\hline F (E) & $9,88^{* *}$ & $20,63^{*}$ \\
\hline Insetos (I) & 1 minuto a 15 minutos & 1 minuto a 120 minutos \\
\hline Um inseto & $0,98 \mathrm{~b}$ & $1,14 \mathrm{c}$ \\
Três insetos & $1,38 \mathrm{a}$ & $1,72 \mathrm{~b}$ \\
Cinco insetos & $1,57 \mathrm{a}$ & $2,05 \mathrm{a}$ \\
\hline F (I) & $15,51^{* *}$ & $25,78^{* *}$ \\
F (E X I) & $3,75^{* *}$ & $3,53^{* *}$ \\
D. M. S. & 0,54 & 0,64 \\
C. V. & 41,08 & 44,25 \\
\hline
\end{tabular}

${ }^{1}$ Médias seguidas da mesma letra não diferem estatisticamente entre si na coluna, pelo teste de Tukey, ao nível de 5\% de probabilidade. (Para análise os dados foram transformados em $\left.(\mathrm{x}+0,5)^{1 / 2}\right)$

TABELA 3 - Valores da análise de desdobramento das interações de cinco espécies versus três densidades de adultos obtidos em genótipos de maracujazeiro referentes ao número de Epicauta atomaria atraídas após a liberação, em teste com chance de escolha. Jaboticabal-SP. 2002-2003.

\begin{tabular}{|c|c|c|c|c|c|c|c|c|c|}
\hline \multicolumn{5}{|c|}{3 minutos } & \multicolumn{5}{|c|}{5 minutos } \\
\hline \multicolumn{5}{|c|}{ Densidades de insetos (I) } & \multicolumn{5}{|c|}{ Densidades de insetos (I) } \\
\hline Espécies (E) & Um & Três & Cinco & F (I D E) & Espécies (E) & Um & Três & Cinco & F (I D E) \\
\hline P. setacea & 0,7 a $\mathrm{B}$ & $0,97 \mathrm{ab}$ & $1,12 \mathrm{ab} A$ & $7,27^{* *}$ & P. setacea & 0,81 a $\mathrm{B}$ & 0,93 ab B & 1,2 a $A$ & 8,4 \\
\hline P. alata & 0,7 a $A$ & $0,81 \mathrm{~b} \mathrm{~A}$ & $0,81 \mathrm{c} \mathrm{A}$ & $0,58^{\mathrm{NS}}$ & P. alata & 0,7 a $A$ & $0,75 \mathrm{~b} \mathrm{~A}$ & 0,86 bc $A$ & $0,82^{\mathrm{NS}}$ \\
\hline P. edulis & 0,86 a $\mathrm{B}$ & $0,84 \mathrm{ab}$ & 1,19 a $\mathrm{A}$ & $6,67^{* *}$ & P. edulis & 0,96 a A & $1,0 \mathrm{ab} A$ & $1,1 \mathrm{ab} A$ & $1,66^{\mathrm{NS}}$ \\
\hline P. cincinnata & 0,81 a $A$ & 1,11 a $A$ & 0,86 bc A & $4,44^{* *}$ & P. cincinnata & 0,75 a $\mathrm{B}$ & 1,2 a $\mathrm{A}$ & 0,96 abc $\mathrm{A}$ & $6,55^{* *}$ \\
\hline P. laurifolia & 0,75 a A & $0,75 \mathrm{~b} \mathrm{~A}$ & $0,75 \mathrm{cA}$ & $0,0^{\mathrm{NS}}$ & P. laurifolia & 0,75 a $\mathrm{A}$ & $0,86 \mathrm{~b} \mathrm{~A}$ & $0,75 \mathrm{c} \mathrm{A}$ & $0,46^{\mathrm{NS}}$ \\
\hline F (E D I) & $0,74^{\mathrm{NS}}$ & $3,46^{\circ *}$ & $6,23^{* *}$ & & F (E D I) & $1,3^{\mathrm{NS}}$ & $3,68^{* *}$ & $6,51^{\circ \mathrm{O}}$ & \\
\hline \multicolumn{5}{|c|}{10 minutos } & \multicolumn{5}{|c|}{15 minutos } \\
\hline \multicolumn{5}{|c|}{ Densidades de insetos (I) } & \multicolumn{5}{|c|}{ Densidades de insetos (I) } \\
\hline Espécies (E) & Um & Três & Cinco & $F($ I D E) & Espécies (E) & Um & Três & Cinco & F (I D GE \\
\hline$P$. setacea & 0,81 a $\mathrm{B}$ & 0,93 ab B & 1,3 a $\mathrm{A}$ & $12,3^{* *}$ & P. setacea & 0,81 a $\mathrm{B}$ & $0,86 \mathrm{ab} \mathrm{C}$ & 1,31 a $\mathrm{A}$ & $9,22^{\circ}$ \\
\hline P. alata & 0,7 a B & $0,75 \mathrm{~b} \mathrm{~A}$ & $0,81 \mathrm{~b} \mathrm{~A}$ & $0,35^{\mathrm{NS}}$ & $P$ alata & 0,7 a A & $0,93 \mathrm{ab} A$ & 0,94 b A & $2,1^{\mathrm{NS}}$ \\
\hline P. edulis & $0,86 \mathrm{a}$ & $0,94 \mathrm{ab} \mathrm{A}$ & $1,14 \mathrm{ab} A$ & $2,7^{\mathrm{NS}}$ & P. edulis & 0,86 a $\mathrm{A}$ & $0,98 \mathrm{ab} \mathrm{A}$ & 0,94 b A & $0,47^{\mathrm{NS}}$ \\
\hline P. cincinnata & 0,86 a $\mathrm{B}$ & 1,1 a $\mathrm{B}$ & $0,89 \mathrm{~b} \mathrm{~A}$ & $4,29^{\mathrm{NS}}$ & P. cincinnata & 0,7 a $\mathrm{B}$ & 1,08 a $A$ & $1,08 \mathrm{ab} \mathrm{A}$ & $5,54^{* \cdots}$ \\
\hline P. laurifolia & 0,7 a A & $0,7 \mathrm{~b} \mathrm{~A}$ & $0,91 \mathrm{~b} \mathrm{~A}$ & $1,90^{\mathrm{NS}}$ & $P$. laurifolia & 0,75 a A & $0,7 \mathrm{~b} \mathrm{~A}$ & 0,75 b A & $0,10^{\mathrm{NS}}$ \\
\hline F (E D I) & $0,82^{\mathrm{NS}}$ & $4,81^{\circ *}$ & $7,3^{* *}$ & & F (E D I) & $0,53^{\mathrm{NS}}$ & $2,34^{\mathrm{NS}}$ & $5,03^{* *}$ & \\
\hline \multicolumn{5}{|c|}{120 minutos } & & & & & \\
\hline \multicolumn{5}{|c|}{ Densidades de insetos (I) } & & & & & \\
\hline Espécies (E) & Um & Três & Cinco & F (I D E) & & & & & \\
\hline P. setacea & 0,96 a $\mathrm{B}$ & 0,91 ab B & $1,49 \mathrm{ab} \mathrm{A}$ & $15,10^{\circ *}$ & & & & & \\
\hline P. alata & 0,7 a $\mathrm{A}$ & $0,7 \mathrm{~b} \mathrm{~A}$ & $0,7 \mathrm{c} \mathrm{A}$ & $0,0^{\mathrm{NS}}$ & & & & & \\
\hline P. edulis & 0,81 a $\mathrm{B}$ & $1,0 \mathrm{ab} \mathrm{AB}$ & 1,12 ab $\mathrm{A}$ & $4,9^{* *}$ & & & & & \\
\hline P. cincinnata & 0,7 a $\mathrm{B}$ & 1,16 a $\mathrm{A}$ & $1,01 \mathrm{bc} \mathrm{A}$ & $7,86^{* *}$ & & & & & \\
\hline P. laurifolia & 0,7 a A & $0,7 \mathrm{~b} \mathrm{~A}$ & $0,75 \mathrm{c} \mathrm{A}$ & $0,13^{\mathrm{NS}}$ & & & & & \\
\hline F (E D I) & $1,88^{\mathrm{NS}}$ & $5,59^{\circ *}$ & $15,20^{* *}$ & & & & & & \\
\hline
\end{tabular}

TABELA 4 - Massa seca de folhas consumida, quando submetida a discos foliares de cinco espécies de maracujazeiro, em três densidades de adultos de Epicauta atomaria, em teste com chance de escolha. Jaboticabal-SP. 2002-2003.

\begin{tabular}{lc}
\hline Espécies (E) & Massa seca de folha consumida $(\mathrm{g})$ \\
\hline$P$. setacea & $0,7098 \mathrm{ab}$ \\
$P$. alata & $0,7084 \mathrm{~b}$ \\
$P$. edulis & $0,7134 \mathrm{a}$ \\
$P$. cincinnata & $0,7088 \mathrm{~b}$ \\
$P$. laurifolia & $0,7071 \mathrm{~b}$ \\
\hline F (E) & $4,47^{*+}$ \\
\hline Insetos (I) & \\
Um inseto & $0,7091 \mathrm{a}$ \\
Três insetos & $0,7096 \mathrm{a}$ \\
Cinco insetos & $0,7099 \mathrm{a}$ \\
\hline F (I) & $0,1841^{\mathrm{NS}}$ \\
F (E X I) & $0,841^{\mathrm{NS}}$ \\
D. M. S. & 0,006 \\
C. V. & 0,84 \\
\hline
\end{tabular}

${ }^{1}$ Médias seguidas da mesma letra não diferem estatisticamente entre si na coluna, pelo teste de Tukey, ao nível de 5\% de probabilidade .(Para análise, os dados foram transformados em $\left.(\mathrm{x}+0,5)^{1 / 2}\right)$. 
TABELA 5 - Número médio de adultos de Epicauta atomaria atraídos por discos foliares de cinco espécies de maracujazeiro, em três densidades de adultos (teste sem chance de escolha). Jaboticabal-SP. 2002-2003.

\begin{tabular}{|c|c|c|c|c|c|c|c|c|}
\hline \multirow[t]{2}{*}{ Espécies (E) } & \multicolumn{8}{|c|}{ Número de insetos atraídos ${ }^{1}$} \\
\hline & 1 minuto & 3 minutos & 5 minutos & 10 minutos & 15 minutos & 30 minutos & 60 minutos & 120 minutos \\
\hline P. setacea & $0,85 \mathrm{ab}$ & $0,93 \mathrm{ab}$ & $1,01 \mathrm{a}$ & $1,04 \mathrm{a}$ & $0,99 \mathrm{a}$ & $1,08 \mathrm{a}$ & $1,01 \mathrm{a}$ & $1,12 \mathrm{a}$ \\
\hline P. alata & $0,77 \mathrm{ab}$ & $0,77 \mathrm{bc}$ & $0,77 \mathrm{c}$ & $0,75 \mathrm{c}$ & $0,86 a b$ & $0,79 \mathrm{~b}$ & $0,79 \mathrm{bc}$ & $0,70 \mathrm{~b}$ \\
\hline P. edulis & $0,87 \mathrm{ab}$ & 0,96 a & $1,04 \mathrm{a}$ & 0,98 a & $0,93 \mathrm{ab}$ & 0,98 a & $0,96 \mathrm{ab}$ & 0,99 a \\
\hline P. cincinnata & 0,95 a & $0,93 \mathrm{ab}$ & $0,97 \mathrm{ab}$ & $0,98 \mathrm{a}$ & $0,95 \mathrm{a}$ & 0,99 a & $1,02 \mathrm{a}$ & 0,96 a \\
\hline P. laurifolia & $0,75 \mathrm{a}$ & $0,75 \mathrm{c}$ & $0,79 \mathrm{bc}$ & $0,77 \mathrm{~b}$ & $0,74 \mathrm{~b}$ & $0,73 \mathrm{~b}$ & $0,74 \mathrm{c}$ & $0,72 \mathrm{c}$ \\
\hline$F(E)$ & $2,71^{*}$ & $4,73^{* *}$ & $6,42^{* *}$ & $6,96^{* *}$ & $3.55^{\circ *}$ & $9,19^{* *}$ & $5,69^{*}$ & $6,55^{* *}$ \\
\hline Insetos (I) & 1 minuto & 3 minutos & 5 minutos & 10 minutos & 15 minutos & 30 minutos & 60 minutos & 120 minutos \\
\hline $\mathrm{Um}$ inseto & $0,74 \mathrm{~b}$ & $0,76 \mathrm{~b}$ & $0,80 \mathrm{~b}$ & $0,78 \mathrm{~b}$ & $0,76 \mathrm{~b}$ & $0,78 \mathrm{~b}$ & $0,77 \mathrm{~b}$ & $0,77 b$ \\
\hline Três insetos & $0,88 \mathrm{a}$ & $0,90 \mathrm{a}$ & $0,95 \mathrm{a}$ & $0,90 \mathrm{ab}$ & $0,91 \mathrm{a}$ & $0,92 \mathrm{a}$ & $0,93 \mathrm{a}$ & $0,89 \mathrm{~b}$ \\
\hline Cinco insetos & $0,89 a$ & $0,94 \mathrm{a}$ & $1,01 \mathrm{a}$ & $1,03 \mathrm{a}$ & $1,01 \mathrm{a}$ & $1,04 \mathrm{a}$ & $1,00 \mathrm{a}$ & $1,03 \mathrm{a}$ \\
\hline $\mathrm{F}(\mathrm{I})$ & $4,90^{* *}$ & $7,15^{\circ *}$ & $7,8^{* *}$ & $6,8 * *$ & $8,80^{* *}$ & $11,06^{* *}$ & $7,5^{* 0}$ & $11,58^{* *}$ \\
\hline$F(E \times I)$ & $0,71^{N S}$ & $2,85^{* *}$ & $2,53^{* *}$ & $2,98^{* *}$ & $2,17^{* *}$ & $1,10^{\mathrm{NS}}$ & $0,59^{\mathrm{NS}}$ & $4,10^{* *}$ \\
\hline D. M. S. & 0,25 & 0,24 & 0,27 & 0,27 & 0,29 & 0,26 & 0,30 & 0,26 \\
\hline C. V. & 30,82 & 28,24 & 29,93 & 30,08 & 32,37 & 28,97 & 33,09 & 28,93 \\
\hline
\end{tabular}

${ }^{1}$ Médias seguidas da mesma letra não diferem estatisticamente entre si, na coluna, pelo teste de Tukey, ao nível de $5 \%$ de probabilidade. (Para análise, os dados foram transformados em $\left.(x+0,5)^{1 / 2}\right) .{ }^{* *}$ Significativo a $1 \%$ de probabilidade. NS Não-significativo.

TABELA 6 - Valores da análise de desdobramento das interações de duas variedades versus duas densidades de adultos obtidos em espécies de maracujazeiro referente ao número de adulto de Epicauta atomaria atraídos após a liberação, em teste sem chance de escolha. Jaboticabal-SP. 2002-2003.

\begin{tabular}{lccl}
\hline Espécies (E) & \multicolumn{3}{c}{ Densidade de insetos (I) } \\
\hline & Um & Dois & F (I D E) \\
P. edulis & $0,96 \mathrm{a} \mathrm{B}$ & $1,19 \mathrm{a} \mathrm{A}$ & $5,81^{\circ}$ \\
P. laurifolia & $0,75 \mathrm{~b} \mathrm{~A}$ & $0,70 \mathrm{~b} \mathrm{~A}$ & $0,30^{\mathrm{NS}}$ \\
\hline F (E D I) & $4,85^{\circ}$ & $26,67^{\circ}$ &
\end{tabular}

${ }^{1}$ Médias seguidas da mesma letra não diferem estatisticamente entre si, na coluna, pelo teste de Tukey, ao nível de $5 \%$ de probabilidade. (Para análise ,os dados foram transformados em $\left.(x+0,5)^{1 / 2}\right){ }^{*}$ Significativo a $5 \%$ de

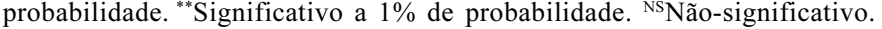

TABELA 7 - Número médio de adultos de Epicauta atomaria atraídos a 1 a 15 minutos e de 1 a 120 minutos, por discos foliares de duas espécies de maracujazeiro, em duas densidades, em teste sem chance de escolha. Jaboticabal-SP. 2002-2003.

\begin{tabular}{lc}
\hline Espécies (E) & Massa seca consumida (g) \\
\hline$P$. edulis & $0,7110 \mathrm{a}$ \\
$P$. laurifolia & $0,7071 \mathrm{~b}$ \\
\hline F (E) & $10,76^{* *+}$ \\
\hline Insetos (I) & $0,7085 \mathrm{a}$ \\
Um inseto & $0,7096 \mathrm{a}$ \\
Dois insetos & $1,00^{\mathrm{NS}}$ \\
F (I) & $1,56^{\mathrm{NS}}$ \\
F (E X I) & 0,038 \\
D. M. S. & 0,52 \\
C. V. & .
\end{tabular}

${ }^{1}$ Médias seguidas da mesma letra não diferem estatisticamente entre si ,na coluna, pelo teste de Tukey ,ao nível de $5 \%$ de probabilidade. (Para análise, ,os dados foram transformados em $\left.(x+0,5)^{1 / 2}\right) .{ }^{* *}$ Significativo a $1 \%$ de probabilidade. Ns Não-significativo.
TABELA 8 - Massa seca de folhas consumida, quando submetida a discos foliares de cinco espécies de maracujazeiro, em três densidades de adultos de Epicauta atomaria, em teste sem chance de escolha. Jaboticabal-SP. 2002-2003.

\begin{tabular}{lc}
\hline Espécies (E) & Massa seca consumida (g) \\
\hline$P$. edulis & $0,7110 \mathrm{a}$ \\
$P$. laurifolia & $0,7071 \mathrm{~b}$ \\
\hline F (E) & $10,76^{* *}$ \\
\hline Insetos (I) & \\
Um inseto & $0,7085 \mathrm{a}$ \\
Dois insetos & $0,7096 \mathrm{a}$ \\
\hline F (I) & $1,00^{\mathrm{NS}}$ \\
F (E X I) & $1,56^{\mathrm{NS}}$ \\
D. M. S. & 0,038 \\
C. V. & 0,52 \\
\hline
\end{tabular}

${ }^{1}$ Médias seguidas da mesma letra não diferem estatisticamente entre si , na coluna, pelo teste de Tukey, ao nível de $5 \%$ de probabilidade. (Para análise, os dados foram transformados em $\left.(\mathrm{x}+0,5)^{1 / 2}\right){ }^{* *}$ Significativo a $1 \% \mathrm{de}$ probabilidade. ${ }^{\mathrm{NS}} \mathrm{Não}$-significativo.

\section{CONCLUSÕES}

1- As espécies $P$. laurifolia e $P$. alata mostraram-se resistentes a $E$. atomaria, expressando o tipo não-preferência para alimentação.

2- As densidades de E. atomaria que melhor discriminam as espécies de maracujazeiro, são três e cinco insetos para o teste com chance de escolha e dois insetos para o teste sem escolha.

\section{REFERÊNCIAS}

AGRIANUAL 2006: anuário da agricultura brasileira. São Paulo: FNP Consultoria \& comércio, 2006. p.370-371.

BALDIN, E.L.L., LARA. F.M. Atratividade e preferência alimentar de adultos de Epicauta atomaria (Germ., 1821)(Col.:Meloidae) em maracujazeiros (Passiflora spp.), sob condições de laboratório. Revista Brasileira de Fruticultura, Jaboticabal, v.24, p.68-71, 2002. 
BAPTISTA, J.Z., BOIÇAJUNIOR, A.L., OLIVEIRA, J.C., JESUS, F.G. Dinâmica populacional de Epicauta atomaria (Germar, 1821)(Coleoptera: Meloidae) em genótipos de maracujazeiro (Passiflora spp.). Ecossistema, Espírito Santo do Pinhal, v.30, 2005.p.71-76.

BOIÇA JUNIOR, A.L. Pragas do maracujá In: RUGGIERO, C. Maracujá: plantio a colheita. Jaboticabal: UNESP, 1998. p.175207.

BOIÇAJUNIOR, A.L., LARA, F.M., OLIVEIRA, J.C., PESSOA, R. Resistência de genótipos de mararacujá a Epicauta atomaria (Germar, 1821) (Coleoptera:Meloidae). Boletin de Sanidad Vegetal, Madrid, v. 22, 2006. p.189-192.

BOARetTo, M,C.A., BRAndÃO, A.L.S. Pragas do maracujazeiro: maracujá produção e mercado. Vitória da Conquista: DFZ/UESB, 1994. p.99-107.
FANCELLI, M. As lagartas desfolhadoras do maracujazeiro. Cruz das Almas: EMBRAPA-CNPMF, 1992.2p.

GALLO, D; NAKANO, O.; SILVEIRANETO, S.; BAPTISTA, G.C.; BERTI FILHO, E.; PARRA, J.R.P.; ZUCCHI, R.A.; ALVES, S.B.; VENDRAMIM, J.D.; MARCHINI, L.C.; LOPES, J.R.S.; OMOTO, S. Entomologia agrícola. Piracicaba: FEALQ, 2002. p. 317-319.

LARA, F.M. Princípios de resistência de plantas a insetos. São Paulo: Ed. Ícone, 1991. p. 235-242.

RODRIGUES NETO, S.M., GUILHERME, D.J. Epicauta atomaria primeiro registro de ocorrência em pomares de maracujá na região oeste do Estado de São Paulo. Arquivos do Instituto Biológico, São Paulo, v.67, n.2, p. 269-270, 2000.

RUGGIERO, C., SÃO JOSÉ, A.R., VOLPE, C.A., OLIVEIRA, J.C. Maracujá para a exportação: aspectos técnicos da produção. Brasília: EMBRAPA-SPI, 19 